\title{
A truly visible vessel in an endoscopic submucosal dissection scare: thinking outside recommendations
}
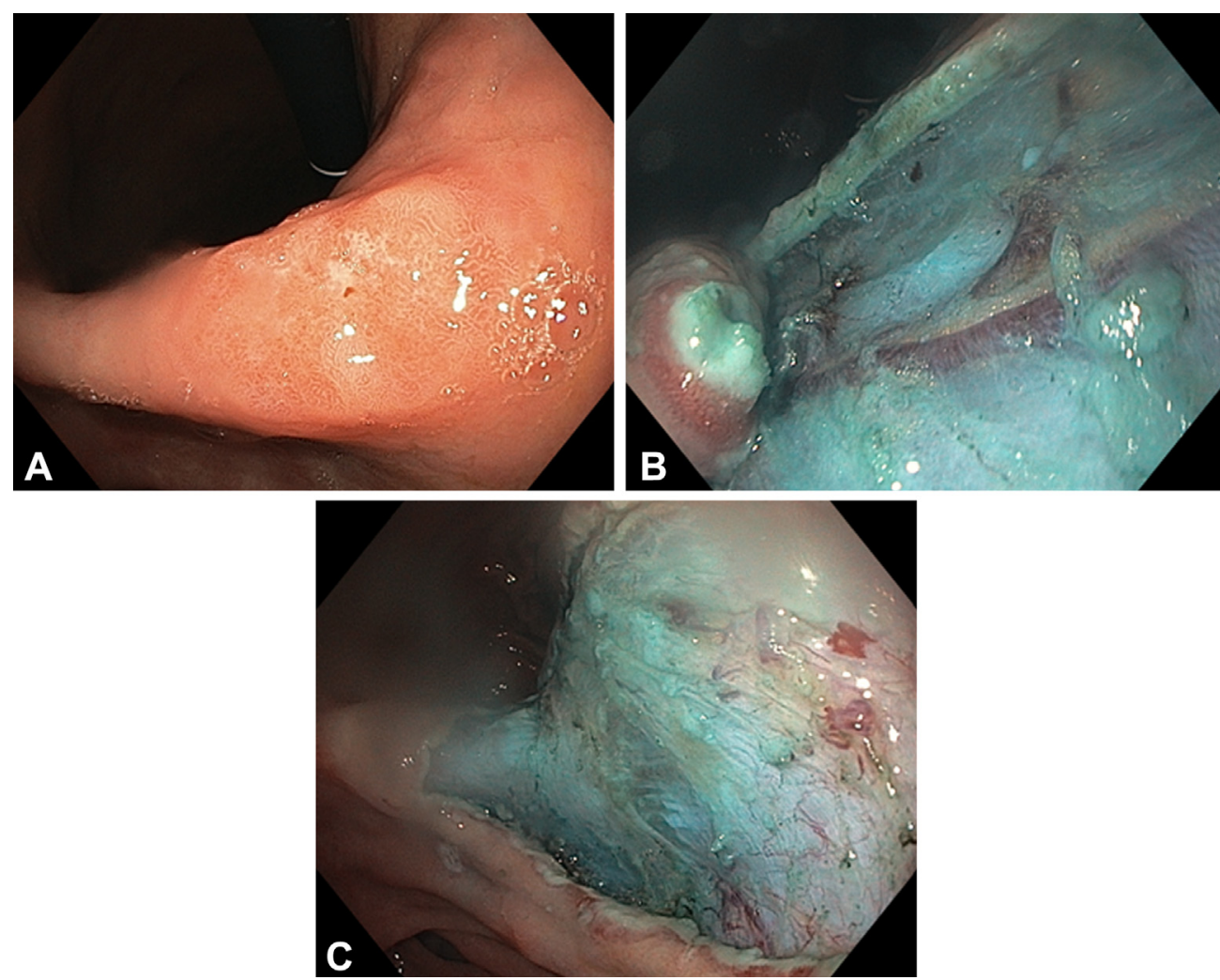

A 75-year-old woman with a superficial lesion type IIa $+\mathrm{C}$ (intramucosal adenocarcinoma) $12 \times 15 \mathrm{~mm}$ in diameter in the incisura angularis (A) was admitted for endoscopic submucosal dissection (ESD). During the procedure, mild bleeding was observed, which was controlled by vessel coagulation by use of the dissection IT knife (Olympus, Tokyo, Japan). Later, in the posterior area, a big vessel, approximately $3 \mathrm{~mm}$ in diameter, was seen (B). The decision was made not to coagulate that vessel immediately, to dissect around the vessel, and to complete en bloc resection. Upon completion of the ESD, a fine layer of submucosa was apparent over the vessel without bleeding (C). Even though the team was aware of the suggestion to coagulate the visible vessels in the artificial ulcer, the decision was not to coagulate that particular vessel, given its big caliber and extension, knowing that if bleeding had occurred, its management would not be straightforward. The patient remained hemodynamically stable. After 1 month of follow-up, no signs of bleeding were noticeable. We estimate that more cases like this occur in daily practice because some patients' clinical situations have not been completely described in previous studies, and thus those cases have no supporting recommendations.

\section{DISCLOSURE}

All autbors disclosed no financial relationships relevant to this publication. 
Mariana Costa, MD, Gastroenterology Department, Centro Hospitalar de Lisboa Central, Lisbon, Portugal, Diogo Libânio, MD, Jorge Lage, MD, Mário DinisRibeiro, MD, PhD, Pedro Pimentel-Nunes, MD, PhD,
Gastroenterology Department, Portuguese Oncology Institute, Porto, Portugal

http://dx.doi.org/10.1016/j.gie.2015.07.030

\section{Commentary}

Gastric cancer is the fourth most common cancer in both sexes worldwide. Early gastric cancer (EGC) is defined as cancer limited to the mucosa or submucosa. The prognosis of EGC is good, with the 5-year gastric cancer-specific survival rate reportedly $99 \%$ if the cancer is resected successfully. Local and less invasive methods to remove the cancer would be indicated in those cancers limited to the mucosa with negligible risk of lymph node metastasis. Most of the time, endoscopic resection is considered curative when submucosal invasion is limited to $500 \mu \mathrm{m}$ in depth. Two endoscopic resection techniques are used to remove EGC, including EMR and endoscopic submucosal dissection (ESD). ESD allows for en bloc complete resection of larger lesions $(>2 \mathrm{~cm})$ and avoids piecemeal resection. This is crucial because the local recurrence rate is $15 \%$ when en bloc resection is not achieved. By achieving en bloc resection, it allows for detailed histopathologic assessment, which is required to confirm curative resection. This procedure helps preserve the stomach and avoid gastrectomy. ESD has replaced EMR for the removal of most gastric lesions. Adverse events associated with endoscopic resection of EGC include bleeding and perforation. Bleeding after ESD can be classified into immediate (during the procedure) or delayed (within 2 weeks or later). More than 76\% of delayed bleeding episodes take place within the first 24 hours after the procedure. Both immediate and delayed bleeding are more common in gastric ESD because of the increased submucosal vessels in this area. In addition, gastric lesions in the middle or upper third of the stomach are at increased risk. For immediate bleeding, electrocautery is used for hemostasis because endoscopic clips interfere with the subsequent resection procedure. Electrocautery can be carried out with different devices. Minor oozing can be controlled by using electrocautery cutting devices such as IT, hook, or dual knife. A critical point in achieving good hemostasis is to identify the exact bleeding point, using water flushing. Prophylactic electrocautery of large submucosal vessels during ESD has been demonstrated to decrease the risk of delayed bleeding up to $60 \%$. For post-ESD coagulation, this is achieved by using hemostatic forceps such as a coagrasper or hot biopsy forceps. In one study, post-ESD coagulation therapy was applied to visible vessels regardless of whether there was any evidence of bleeding at the end of ESD. The study demonstrates that prophylactic treatment of visible vessels appears to be an important factor for prevention of delayed bleeding after ESD. In the case presented, the authors decided not to perform prophylactic coagulation of the visible vessel after the ESD. On the basis of current literature and guidelines, it is suggested that prophylactic therapy leads to a decreased risk of bleeding later on. Additional studies need to be performed to confirm the finding of the current case, particularly for challenging patients who are receiving long-term anticoagulant agents.

Wei-Chung Chen, MD Fellow in Gastroenterology \& Hepatology Mayo Clinic Florida

Massimo Raimondo, MD Associate Editor for Focal Points

\section{Direct digital cholangioscopic visualization of suture causing a biliary anastomotic stricture}

A 61-year-old man underwent orthotopic liver transplantation for end-stage liver disease and cirrhosis resulting from alcohol abuse. He did well in the immediate postoperative period, but 4 weeks after transplantation he experienced jaundice and elevated liver chemistry determinations with a cholestatic pattern. ERCP demonstrated a tight anastomotic stricture with donor intrahepatic and extrahepatic ductal dilatation. An $8.5 \mathrm{~F}$ plastic biliary stent was placed, with improvement in his liver chemistry determinations and resolution of jaundice. On repeated ERCP, there was decreased intrahepatic ductal dilatation, but the anastomotic stricture persisted (A). The Spyglass digital cholangioscope (Boston Scientific, Natick, Mass) was used to examine the stenosis. Cholangiographically, 